\title{
LAS REDES CONCEPTUALES COMO INSTRUMENTO PARA EVALUAR EL NIVEL DE APRENDIZAJE CONCEPTUAL DE LOS ALUMNOS. UN EJEMPLO PARA EL TEMA DE DINÁMICA
}

\author{
CILIBERTI, N. ${ }^{1}$ y GALAGOVSKY, L.R. ${ }^{2}$ \\ ${ }^{1}$ Instituto San Roque. Plaza 1160. 1427 Buenos Aires. Argentina. \\ ${ }^{2}$ Centro de Formación e Investigación en Enseñanza de las Ciencias, Facultad de Ciencias Exactas \\ y Naturales. Universidad de Buenos Aires. Ciudad Universitaria. Pabellón II. 1428 Buenos Aires, \\ Argentina.
}

\begin{abstract}
SUMMARY
Since the first articles concerning Concept Mapping many more have been reported looking for instruments to evaluate the level of student's meaningful learning.

In the present work we show that the construction of nuclear sentences for Concept Nets (Galagovsky, 1993a, b, 1996; Galagovsky and Ciliberti, 1994) demands high level of semantic analysis, which seems to help in inducing conceptual change. Results suggest that this type of class experience may help teachers to get complementary information about his/her student's meaningful learning level.
\end{abstract}

\section{INTRODUCCIÓN}

Las recientes reformas de los sistemas educativos de varios países de habla hispana han contribuido, de forma especial, a la comunicación entre grupos de investigación en aprendizaje y docentes que buscan, con renovado interés, estrategias alternativas de enseñanza.

Se han publicado en estos últimos años muchos trabajos acerca de la utilización de mapas conceptuales (Novak, 1988; Ontoria, 1995) y mapas semánticos (Heimlich, 1991) en situaciones de enseñanza y aprendizaje.

A partir de los trabajos de investigación que se derivan de la descripción de las redes conceptuales (Galagovsky, 1993a, b, 1996), encontramos que las exigencias semánticas requeridas para la confección de estos instrumentos obligan a su constructor a realizar un análisis verdadera- mente conceptual del contenido. Este nivel es claramente diferente del nivel semántico ambiguo $-\mathrm{y}$ muchas veces superficial- requerido para la construcción de los mapas.

En el presente artículo relatamos una experiencia de aula, que nos mostró que la confección de mapas es más sencilla que la construcción de redes conceptuales, pero que el esfuerzo de comprensión conceptual exigido para la confección de las oraciones nucleares de la red es un marco de trabajo que ayuda a dar significación al aprendizaje.

Asimismo, resultó interesante confrontar la información que brindó este tipo de actividad, con la información codificada en las calificaciones obtenidas por los alumnos en una evaluación tradicional. 


\section{OBJETIVOS DEL TRABAJO}

La intención que motivó este trabajo fue la de realizar una experiencia de aula que permitiera:

a) indagar sobre las diferentes exigencias intelectuales que implica para los alumnos la construcción de mapas (Heimlich, 1991; Novak, 1988; Ontoria, 1995) o de redes conceptuales (Galagovsky, 1996);

b) analizar la relación entre las calificaciones obtenidas por los alumnos y su habilidad para la confección de redes conceptuales.

Cabe aclarar que, por ser una experiencia de aula, no se pudieron extremar las condiciones experimentales que exige una investigación rigurosa sobre el tema (Colás, 1994); sin embargo, los resultados obtenidos muestran que el propio docente, desde su rol, puede organizar estrategias indagatorias que producen interesantes resultados.

\section{CONSIDERACIONES TEÓRICAS: DIFE- RENCIAS ENTRE MAPAS Y REDES CONCEPTUALES}

Antes de describir la experiencia es oportuno recordar similitudes y diferencias entre los mapas y las redes conceptuales.

Ambos instrumentos tienen un aspecto gráfico y un aspecto semántico.

Con respecto al aspecto gráfico, puede decirse que los mapas y las redes conceptuales se parecen. De hecho, ambos instrumentos están formados por nodos (es decir, palabras enmarcadas) y conexiones entre nodos (es decir, líneas que unen los nodos y que dan cuenta de que entre ellos existe alguna relación).

Una diferencia importante es que los mapas conceptuales tienen jerarquía gráfica (Novak, 1988, 1991; Ontoria, 1995); es decir, los conceptos más abarcativos se

Cuadro I

Diferencias fundamentales entre los aspectos semántico y gráfico necesarios para la construcción de un mapa o de una red conceptual.

\begin{tabular}{|c|c|c|}
\hline & Mapa conceptual & Red conceptual \\
\hline Nodos & $\begin{array}{l}\text { Se completan con sustantivos, } \\
\text { verbos o adjetivos. } \\
\text { Se admiten expresiones matemáticas. }\end{array}$ & $\begin{array}{l}\text { Se completan con sustantivos o sustantivos+adjetivos, } \\
\text { que sean conceptos relevantes del tema. } \\
\text { La repetición de nodos está prohibida. } \\
\text { No se aceptan fórmulas matemáticas, } \\
\text { excepto unidas por la leyenda } \\
\text { «se simboliza mediante». }\end{array}$ \\
\hline $\begin{array}{l}\text { Leyendas } \\
\text { que unen } \\
\text { los nodos }\end{array}$ & $\begin{array}{l}\text { Se utiliza cualquier clase de palabras } \\
\text { para formar proposiciones entre nodos. } \\
\text { La extensión de una oración } \\
\text { puede abarcar más de dos } \\
\text { nodos consecutivos. }\end{array}$ & $\begin{array}{l}\text { Se utilizan palabras y verbos muy precisos } \\
\text { que completan una oración nuclear } \\
\text { de óptimo significado entre dos } \\
\text { nodos consecutivos (Galagovsky, 1993a, 1996) }{ }^{1} \text {. } \\
\text { La oración nuclear no puede extenderse } \\
\text { consecutivamente a más de dos nodos. } \\
\text { Las oraciones nucleares } \\
\text { se leerán siguiendo el recorrido de una flecha. }\end{array}$ \\
\hline $\begin{array}{l}\text { Jerarquía } \\
\text { gráfica }\end{array}$ & $\begin{array}{l}\text { Es absolutamente necesaria. } \\
\text { Esta jerarquía vertical debe } \\
\text { reflejar la jerarquía conceptual } \\
\text { específica del tema. }\end{array}$ & $\begin{array}{l}\text { No es necesaria. Los conceptos más } \\
\text { importantes son los más relacionados. }\end{array}$ \\
\hline
\end{tabular}


explicitan en la parte superior del mismo y, descendiendo por el mapa, se encuentran los conceptos de jerarquía intermedia y luego los menos abarcativos. La lectura de un mapa conceptual es, entonces, de arriba hacia abajo.

Las redes conceptuales, en cambio, no requieren jerarquía gráfica vertical; por lo tanto, las conexiones entre nodos, en vez de líneas, son flechas que orientan el sentido de la lectura (Galagovsky, 1993a, b, 1996; Galagovsky y Ciliberti, 1994).

Es en el aspecto semántico donde los mapas y las redes se diferencian fundamentalmente. Es decir, importa con qué tipo de palabras está permitido llenar los nodos y completar las leyendas sobre los nexos.

En el cuadro I se muestran en forma esquemática las diferencias fundamentales entre un mapa y una red conceptual.

Otra diferencia fundamental es que los mapas conceptuales se utilizan para la investigación del aprendizaje individual e idiosincrático; en cambio, las redes conceptuales se plantean, preferentemente, como un instrumento didáctico.

Los mapas semánticos (Heimlich, 1991) permiten total libertad gráfica y semántica.

\section{RELATO DE LA EXPERIENCIA}

Nuestro trabajo con redes conceptuales en situación de aula (Ciliberti, 1994) nos había proporcionado fuertes indicios acerca de que la construcción de mapas (semánticos o conceptuales) requiere de los alumnos menor esfuerzo intelectual que la construcción de redes conceptuales para el mismo contenido conceptual. Estas observaciones se evidenciaban, particulamente, en que los mapas admiten en su formato la inclusión de expresiones semánticas en proposiciones, de tal forma que una oración completa puede atravesar varios nodos consecutivos (Novak, 1991). Pudimos observar que los instrumentos así confeccionados no reflejaban necesariamente un aprendizaje significativo y, muchas veces, los mapas producidos por los alumnos eran definiciones aprendidas memorísticamente escritas en forma vertical, con algunas palabras enmarcadas.

\section{Diseño de la experiencia}

La experiencia se llevó a cabo con un curso mixto de 37 alumnos de $4^{\circ}$ año de bachillerato de un instituto privado confesional.

Luego de haberse enseñado el tema de Dinámica (Chebli, 1984; Resnik y Halliday, 1984; Tipler, 1984; Tricárico y Bazo, 1994; Castiglioni, Perazzo y Rela, 1994), la consigna fue efectuar, por grupos, una red conceptual del tema.
La actividad comenzó con la libre agrupación de 4, 5 ó 6 alumnos, según afinidad o amistad. Dos alumnas que generalmente trabajaban aisladas y tenían dificultades para integrarse al resto de los alumnos no lograron participar en ningún grupo y hubieron de trabajar juntas con el fin de cumplir con la consigna.

Una vez agrupados, se solicitó a los alumnos que escribieran una lista con los conceptos relevantes aprendidos en Dinámica y que luego los relacionaran mediante nexos, formando las oraciones nucleares de la red conceptual sin intervención docente alguna. Se destinaron 80 minutos para efectuar la consigna y se les permitió consultar libros o apuntes de clase. Al finalizar los 80 minutos, el instrumento confeccionado por cada grupo fue recogido por la docente y se avisó a todos que se tomaría una evaluación la clase siguiente.

En la clase siguiente se tomó la evaluación prevista. El instrumento evaluativo utilizado se muestra en el anexo I. El tipo de preguntas allí expuestas es similar a las trabajadas durante las clases previas, en la resolución de problemas.

A los efectos de analizar las habilidades de cada grupo en la confección de la red -y en coherencia con los objetivos- no se realizó una red conceptual consensuada conjuntamente con toda la clase y la docente. Esta red hubiera sido la que muestra el anexo II.

\section{Condiciones de la experiencia}

Para poder indagar sobre nuestros objetivos debimos tener en cuenta los siguientes aspectos:

\section{1) Los alumnos ya sabían construir redes conceptuales:}

La técnica de construcción de redes conceptuales era bien conocida por los alumnos del cuarto año de bachillerato, quienes ya habían confeccionado dos redes para el tema de Cinemática. Estas construcciones habían sido efectuadas en actividades de toda la clase con la conducción de la docente. Los pasos de estas actividades habían sido (Galagovsky y Ciliberti, 1994):

a) Confección de la lista de conceptos en el pizarrón.

b) Construcción de las oraciones que vinculan pares de conceptos. Los alumnos tienen un tiempo para pensar en pares de conceptos y sus posibles vinculaciones; luego, se escriben las opciones en el pizarrón y se discute hasta arribar a la mejor opción semántica.

c) Una vez completa y consensuada, la red es copiada en las carpetas de los alumnos.

2) Se evitó, deliberadamente, la elaboración conjunta de la Red (Anexo II).

Nuestra experiencia nos indicaba que muchos alumnos hacen mapas en lugar de redes conceptuales y sólo toman conciencia de esa diferencia durante la puesta en 


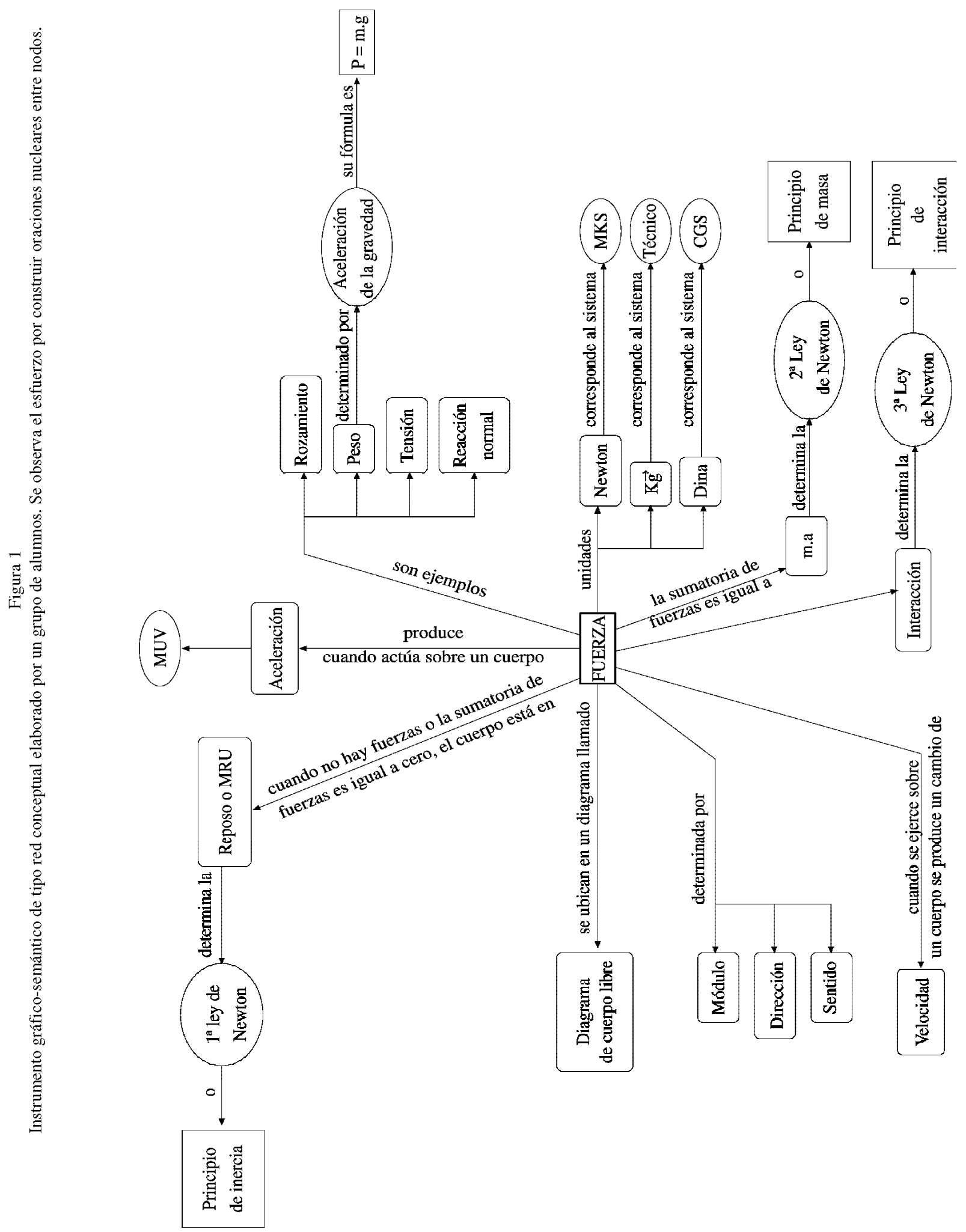




\section{INVESTIGACIÓN DIDÁCTICA}

común final, cuando se discute entre todos y con el docente, las opciones semánticas alternativas para la construcción de las oraciones nucleares de la red.

Esa puesta en común con toda la clase, con intervención del docente, como actividad de cierre de un tema, es un momento enriquecedor, por cuanto es entonces cuando los alumnos pueden realizar la reflexión metacognitiva sobre el contenido conceptual aprendido y encontrar las oraciones nucleares que expresan semánticamente con mayor precisión la trama conceptual de dicho contenido.

\section{RESULTADOS OBTENIDOS}

\section{Tipos de instrumentos gráfico-semánticos con- feccionados por los alumnos}

Ante la consigna de construir la red conceptual, cada grupo confeccionó un diagrama gráfico-semántico. Estos diagramas pueden agruparse en dos categorías: llamamos diseño tipo red a aquellos instrumentos que pueden reconocerse como redes conceptuales, en los cuales se respetó la consigna de «hacer oraciones nu-

Figura 2

Mapa 1: Ejemplo de instrumento gráfico-semántico construido por un grupo de alumnos.

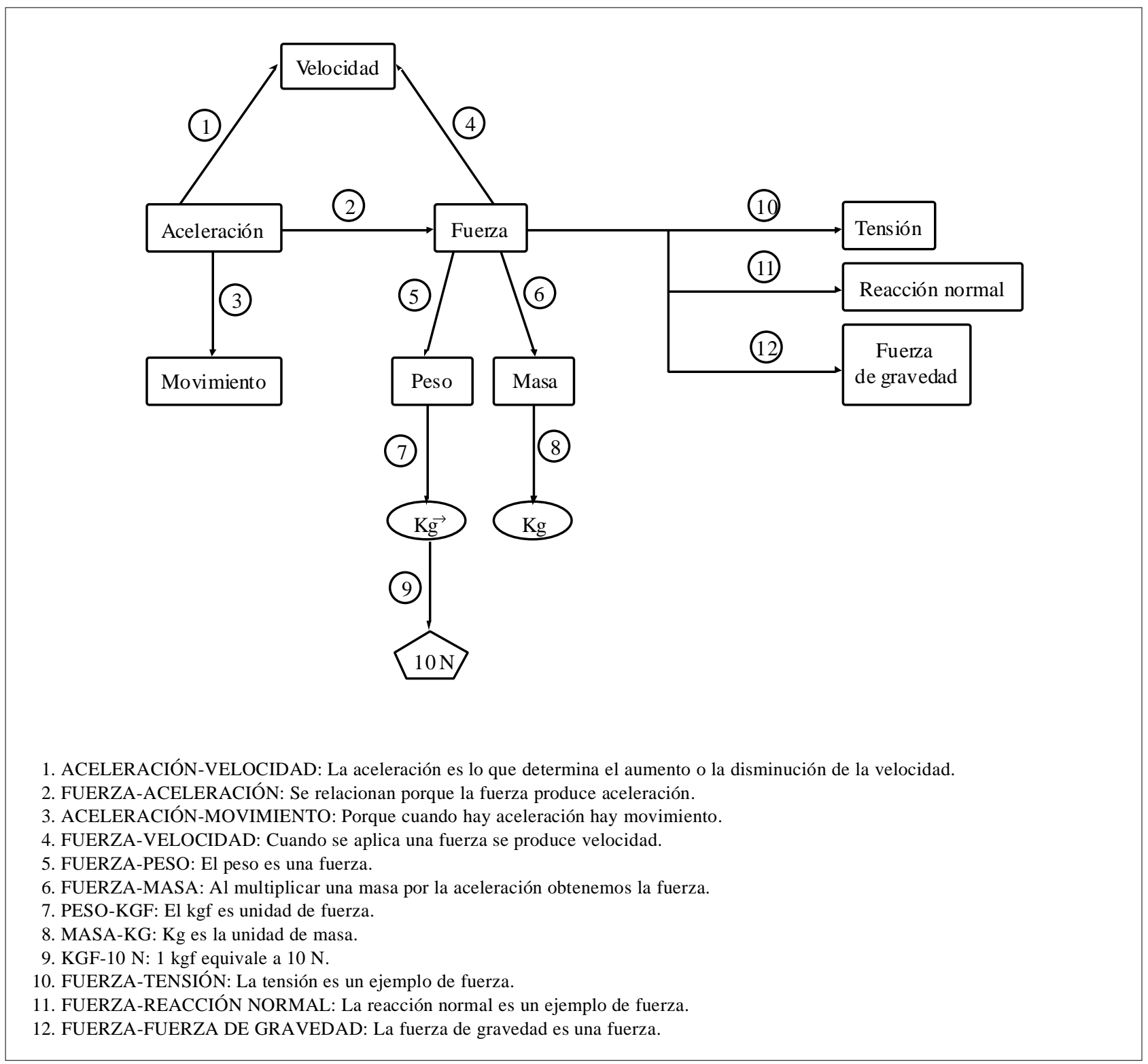


Cuadro II

Comparación entre la red conceptual del anexo II y el instrumento gráfico de la figura 1.

\begin{tabular}{|c|c|c|}
\hline & Red del anexo II & Red de la figura 1 \\
\hline$a$ & $\begin{array}{l}\text { Existen tres conceptos principales } \\
\text { (más relacionados): fuerza, fuerza } \\
\text { resultante y aceleración. } \\
\text { Las oraciones que describen el concepto } \\
\text { de fuerza están unidas directamente a él; } \\
\text { las oraciones que vinculan los conceptos } \\
\text { propios de dinámica están conectados } \\
\text { al nodo fuerza resultante. }\end{array}$ & $\begin{array}{l}\text { Utiliza como nodo central el concepto de fuerza. No aparece el concepto } \\
\text { de fuerza resultante como nodo, si bien este concepto está } \\
\text { presente en forma subsumida en las dos leyendas en que } \\
\text { se habla de «sumatoria de fuerzas». }\end{array}$ \\
\hline$b$ & $\begin{array}{l}\text { No aparece el concepto de velocidad, } \\
\text { que era propio del tema anterior } \\
\text { (cinemática). }\end{array}$ & $\begin{array}{l}\text { Aparece el concepto de velocidad correctamente vinculado a fuerza. } \\
\text { Esta vinculación revelaría que los alumnos asocian la aplicación de una } \\
\text { fuerza con el cambio de movimiento, pero no está claro si ellos creen } \\
\text { necesaria la aplicación de una fuerza (distinto de cero) en el } \\
\text { caso del MRU. (Parece que los alumnos no consideran el circuito } \\
\text { conceptual de } \Sigma F=0 \text { de la red del anexo II). }\end{array}$ \\
\hline$c$ & $\begin{array}{l}\text { El concepto de cuerpo está conectado } \\
\text { al de masa cerrando un circuito } \\
\text { conceptual que conecta los conceptos } \\
\text { de fuerza resultante y aceleración. }\end{array}$ & $\begin{array}{l}\text { La vinculación entre fuerza y aceleración es correcta; sin embargo, } \\
\text { al estar ausentes los conceptos de cuerpo y masa como nodos, } \\
\text { no se visualiza el circuito conceptual que los uniría (ver red anexo II). }\end{array}$ \\
\hline$d$ & $\begin{array}{l}\text { Nuevamente los conceptos de cuerpo y masa } \\
\text { forman parte de un circuito conceptual } \\
\text { que conecta -y al mismo tiempo diferencia- } \\
\text { entre principio de interacció y } \\
\text { principio de masa. }\end{array}$ & $\begin{array}{l}\text { La ausencia de los conceptos de cuerpo y masa impide una vinculación } \\
\text { conceptual entre los nodos principio de interacción y principio de masa. }\end{array}$ \\
\hline$e$ & $\begin{array}{l}\text { El concepto de interacción está sobre } \\
\text { la leyenda que une cuerpo con } \\
\text { principio de interacción. }\end{array}$ & $\begin{array}{l}\text { Interacción es un nodo, pero su vinculación con fuerza } \\
\text { no ha podido ser expresada (posiblemente, debido a lo explicado en } d \text { ). }\end{array}$ \\
\hline$f$ & $\begin{array}{l}\text { Los nodos de los tres principios } \\
\text { pudieron haberse relacionado } \\
\text { explícitamente con cada ley de Newton. }\end{array}$ & $\begin{array}{l}\text { Las flechas sobre las que se escribió una o, deberían reemplazarse por un } \\
\text { «es también conocido como» para poder formar una oración nuclear. }\end{array}$ \\
\hline$g$ & $\begin{array}{l}\text { Del nodo peso salen dos relaciones que } \\
\text { lo caracterizan. }\end{array}$ & $\begin{array}{l}\text { Del nodo peso sale una relación correcta; pero del nodo aceleración } \\
\text { de la gravedad sale una relación equivocada, que debió haber } \\
\text { estado conectada a peso. }\end{array}$ \\
\hline
\end{tabular}

cleares entre nodos»; y denominamos diseño tipo mapa a aquéllos otros en los que no se respetó dicha consigna.

En la figura 1 se muestra un diseño tipo red conceptual; y en las figuras 2 y 3 se muestran dos ejemplos de diseños tipo mapa (los originales manuscritos han sido transcriptos en ordenador para mayor claridad en la lectura). En el anexo II se muestra la red conceptual sobre la que se basó la enseñanza del tema y que hubiera podido quedar plasmada en el pizarrón y consensuada por toda la clase. 
Figura 3

Mapa 2: Construido por dos alumnos.

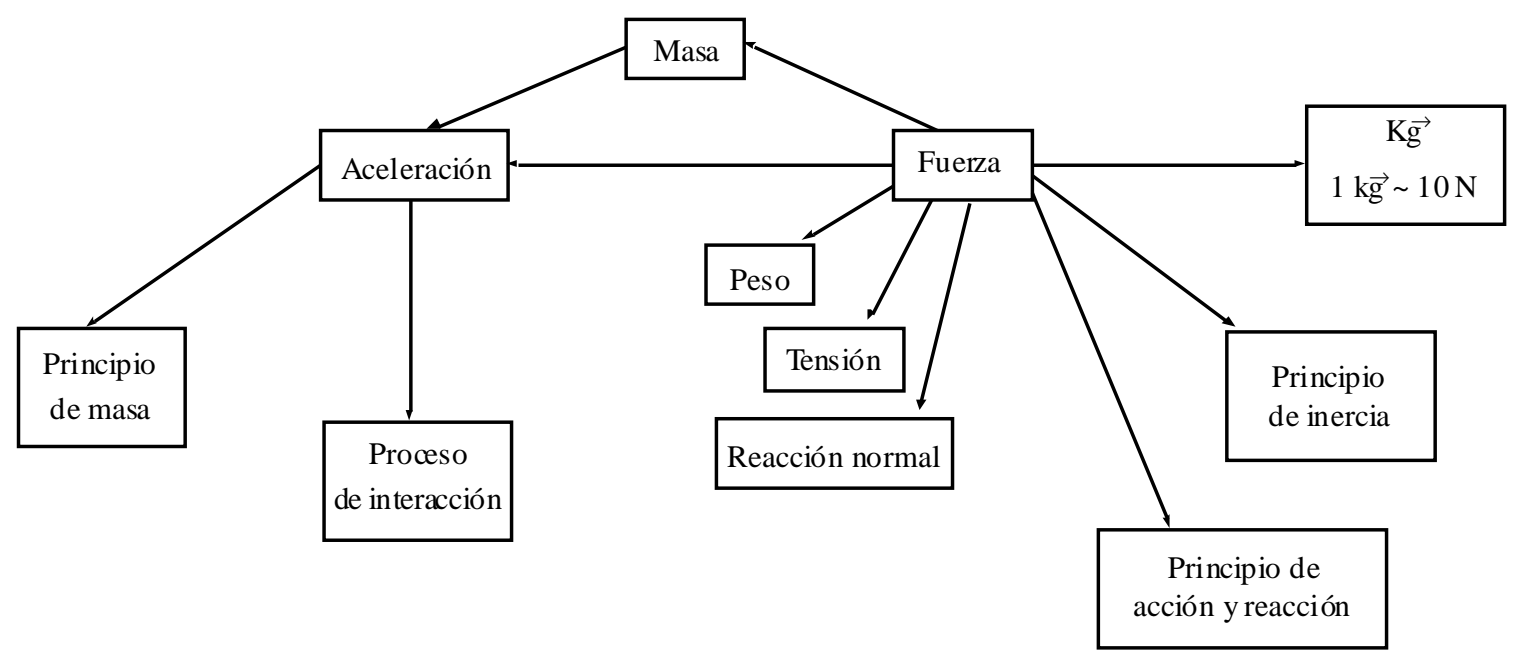

* PROCESO DE INTERACCIÓN: Cuando dos cuerpos interactúan.

* PRINCIPIO DE INERCIA ( $1^{\text {a }}$ ley de Newton): Un cuerpo se encuentra en reposo cuando no actúan fuerzas sobre él o actúan varias que se anulan entre sí.

* PESO: Fuerza que ejerce la Tierra sobre los objetos.

* 1 KGF: Peso del kg patrón, 1 kgf: 10 N.

* FUERZA DE ROZAMIENTO: Fuerza que se opone al movimiento.

* REACCIÓN NORMAL: Fuerza que ejerce el plano sobre el cuerpo.

* TENSIÓN: Fuerza que ejerce una soga sobre el cuerpo.

* PRINCIPIO DE MASA ( $2^{\mathrm{a}}$ ley de Newton): La aceleración que adquiere un cuerpo es proporcional y tiene igual dirección y sentido que la fuerza aplicada.

* NEWTON (N): Unidad de fuerza.

* PRINCIPIO DE ACCIÓN Y REACCIÓN: ( $3^{\mathrm{a}}$ ley de Newton): Si un cuerpo aplica una fuerza sobre otro cuerpo, éste ejerce sobre el primero una fuerza con el mismo módulo y dirección pero con sentido contrario.

* ACELERACIÓN: Cuando se aplica una fuerza al cuerpo.

\section{Análisis del instrumento gráfico cuyo diseño fue de tipo red conceptual}

Cinco grupos presentaron diseños de este tipo, con mayor o menor cantidad de relaciones o de aspectos clasificatorios.

En la figura 1 se muestra un ejemplo representativo que, más allá de la ubicación gráfica de los nodos, es muy coherente con la red del anexo II.

Algunas observaciones que surgen de dicha comparación se muestran en el cuadro II.

\section{Análisis del instrumento denominado mapa 1}

En estos casos no se presentó el formato exigido desde la consigna. Tres grupos de alumnos fueron los que no alcanzaron a cumplir con la consigna de armar oraciones nucleares entre nodos. En la figura 2 se muestra un ejemplo denominado mapa 1 , representativo de este grupo. En él, los alumnos numeraron las flechas conectoras y escribieron debajo del gráfico las oraciones que justificarían la conexión gráfica entre dos conceptos; sin embargo, más allá de los errores que señalaremos a continuación, se observa que muchas de esas oraciones no podrían transcribirse en el lugar indicado leyendo el sentido indicado por las flechas (el caso de las oraciones numeradas como $2,3,6,7,10,11$ y 12).

Si se analizan las oraciones escritas en el ejemplo del mapa 1 se pueden hacer las siguientes observaciones:

a) Hay una inversión en la lógica de la causalidad en la oración 1.

b) No se distinguen las posibilidades de $\Sigma F$ (sumatoria de fuerzas) $=0$ o $\Sigma F=$ distinto de cero. Parecería 
asumirse que la sumatoria de fuerzas siempre tiene valores distintos de cero.

c) La omisión conceptual señalada en el ítem $b$, se pondría de manifiesto en las relaciones incorrectas establecidas para la oración 3 entre aceleración y movimiento, donde no se especifica el tipo de movimiento adquirido.

(Cabe señalar que sólo se estudiaron situaciones cuyas fuerzas eran constantes).

d) Error conceptual en la oración 4, donde se asocia fuerza con velocidad. Este error podría estar relacionado también con la omisión conceptual señalada en el ítem $b$.

e) Las oraciones 10,11 y 12 podrían incluirse como «son ejemplo de»-invirtiendo el sentido de la flecha para su lectura correcta-. Sin embargo, el nodo peso no está contemplado en esta clasificación y sí lo está la fuerza de gravedad. Entendemos que es probable que estos alumnos no hubieran comprendido totalmente el concepto peso y su diferenciación con el concepto masa. Esta última afirmación estaría avalada, también, por la disposición gráfica paralela con que están ubicados peso y masa, conceptos que sólo se diferencian en el mapa por sus respectivas unidades (Anexo II).

Estos grupos no llegaron a escribir las oraciones nucleares dentro de la red, aunque se esforzaron por hacerlo.

Esta etapa intermedia entre hacer la lista de conceptos y tratar de vincularlos en un instrumento gráfico-semántico insume tiempo e induce a armar argumentaciones conceptuales alternativas hasta alcanzar la opción semántica que mejor convenza al grupo.

El pasaje de un instrumento como el mapa 1 hasta uno tipo red como el de la figura 1 demanda aún discusiones y ajustes.

«En la actividad de puesta en común final con el docente es donde se organizan definitiva y consensuadamente las oraciones nucleares que expresan las relaciones entre pares de nodos consecutivos con una forma semántica precisa de máximo significado.» Un paso crítico de esta puesta en común es aquél en que cada grupo explicita las vinculaciones que parten de un concepto de «la red» que ha construido; el docente puede registrar esas alternativas en la pizarra para que todos los grupos las tengan a la vista y se pueda debatir cuál es la alternativa óptima. En esta argumentación de aceptación o descarte de formas semánticas y vinculaciones conceptuales es donde se hacen evidentes los errores y las preconcepciones. Lo importante es que estas concepciones erróneas se hacen evidentes no sólo para el docente, sino también para la mayoría de los alumnos.

Esta actividad de puesta en común también requiere tiempo; no es tiempo que se «pierde», es tiempo que se invierte en impulsar el cambio conceptual de los alumnos.

\section{Análisis del intrumento gráfico denominado mapa 2}

En el mapa 2 de la figura 3 se muestra el trabajo realizado por el grupo de las dos alumnas mencionadas en un párrafo anterior. En él se observa que ni siquiera se intentó ubicar leyendas sobre las flechas.

Este grupo entregó el mapa 2 conjuntamente con un resumen de lo aprendido -según se muestra en la figura 3 -, formado principalmente por definiciones.

Analizando esta presentación pueden hacerse los siguientes señalamientos:

a) Las definiciones de la lista-resumen (párrafo inferior de la figura 3) no están numeradas, lo que induce a suponer que hubo una total falta de intención de cumplir con la consigna de la tarea. Dichas definiciones no se corresponden, además, con pares de conceptos relacionados en el gráfico, de tal forma que no es posible incluirlas en las flechas.

b) No está claro el concepto de proceso de interacción.

c) En la redacción del principio de inercia (segunda definición) no se hace referencia al movimiento rectilíneo uniforme (MRU). Si asumimos que esta omisión es debida a la falta de comprensión conceptual del principio, entonces podríamos inferir la lógica omisión de nodos como movimiento o velocidad en el mapa.

d) Ninguna de las oraciones o definiciones del resumen explicitan las relaciones entre los conceptos de masa y aceleración y fuerza y masa que están vinculados mediante flechas en el gráfico.

La actitud tradicional de estas alumnas durante las clases del año lectivo era pasiva e individualista, aunque con prolijidad y cumplimiento de las actividades propuestas. Entendemos que es muy significativo que estas dos alumnas no hayan podido cumplir con la consigna de hacer una red con sus oraciones nucleares. Como dijimos antes, esta consigna requiere interacción entre los alumnos en la explicitación de sus ideas e hipótesis y la argumentación con su correspondiente dosis de participación activa.

El instrumento presentado por las alumnas parece un claro ejemplo de falta de interés en cumplir la consigna; sin embargo, dada la buena disposición general de estas adolescentes en cumplir con las tareas escolares, consideramos que es apropiado deducir una dificultad intelectual más que una desidia actitudinal frente a la propuesta.

En otras palabras, de la impresión de que a estas alumnas les fue mucho más sencillo evocar textos memorizados que revisar metacognitivamente los conocimientos «alojados» en sus estructuras cognitivas.

Entendemos que el instrumento gráfico presentado, de tipo mapa no les ha exigido esta revisión metacogni- 
tiva que se requiere para llegar a confeccionar una red conceptual.

A su vez, se hace evidente nuestra dificultad para apreciar el nivel de aprendizaje significativo de estas alumnas analizando el gráfico y el resumen que entregaron. Esta dificultad ya ha sido relatada por Ontoria (1995) cuando menciona que el docente encuentra limitaciones al querer indagar sobre el manejo conceptual profundo de sus alumnos y, habiendo solicitado a éstos que confeccionen un mapa conceptual, requieren realizar una entrevista personal posterior -con cada uno- para sondear las implicaciones asociadas a las omisiones o a las relaciones no explícitas del mapa.

\section{Datos de la evaluación}

A la semana siguiente de haber recogido los instrumentos confeccionados por los alumnos y sin devolvérselos, se tomó la evaluación que se muestra en el anexo I, según se había avisado.

Los dos problemas de la evaluación fueron similares a los trabajados durante las clases del tema.

En la tabla I se muestra la relación entre las calificaciones obtenidas por los alumnos en la evaluación y las categorías de diseño de redes conceptuales o mapas, según las pautas señaladas anteriormente. La calificación máxima posible para la evaluación era de 10 puntos y la aprobación mínima de 6 puntos.

El análisis de los resultados nos permitó hacer interesantes inferencias y confirmar las hipótesis que nos habíamos planteados, según se detalla a continuación.

\section{ANÁLISIS DE LA RELACIÓN ENTRE LAS CALIFICACIONES Y LAS HABILIDADES PARA CONSTRUIR MAPAS O REDES CONCEPTUALES}

Los alumnos que presentaron instrumentos gráficosemánticos clasificados como diseño tipo red aprobaron la evaluación tradicional en un $80 \%$ (Tabla I). Los casos de desaprobación - prácticamente un alumno por grupopueden interpretarse como el resultado de la incorporación de «amigos» que no tenían un nivel de aprendizaje homogéneo con el resto ${ }^{2}$. Como consecuencia, suponemos que difícilmente estos alumnos que reprobaron hubieran participado activamente en las discusiones grupales para la confección de las oraciones nucleares.

Es interesante destacar que los diferentes grupos que integran esta categoría presentaron redes de mayor o menor grado de complejidad, con algunas imprecisiones en las oraciones nucleares, pero prácticamente con escasos errores conceptuales.

Los grupos de alumnos que presentaron instrumentos clasificados como diseño tipo mapa 1 resultaron ubicar- se en una franja de calificaciones donde sólo el $50 \%$ ha aprobado. En sus instrumentos faltaban incluir conceptos o las oraciones propuestas tenían poca claridad conceptual, o dejaban ver errores derivados, generalmente, de concepciones aristotélicas.

Las dos alumnas que no intentaron o no pudieron escribir las oraciones nucleares obtuvieron, sin embargo, buenas notas en la evaluación. Este hecho podemos interpretarlo como una respuestan proveniente de aprendizajes de estilo memorístico; es decir, que es probable que estas alumnas pudieran reproducir algoritmos de resolución de problemas, esquemas y reglas para responder a problemas convencionales, pero no podemos evaluar fehacientemente si en ellas se ha producido un cambio conceptual o un aprendizaje significativo.

\section{CONCLUSIONES}

Diferencia entre las exigencias intelectuales que implica la construcción de mapas o redes conceptuales

Dado que todos los alumnos conocían la técnica de confección de redes conceptuales y teniendo en cuenta el hecho de que algunos grupos no pudieron configurar las oraciones nucleares entre nodos, pero sí esbozaron oraciones más o menos ambiguas o definiciones, consideramos que es válido concluir que la explicitación y el ajuste de dichas oraciones nucleares entre nodos implica una mayor dificultad intelectual, que requiere una revisión semántica de los aspectos conceptuales del tema.

Estimamos que muchos alumnos de los que confeccionaron diseños tipo mapa 1 hubieran podido mejorar su nivel de aprendizaje conceptual durante las discusiones que se originan en la puesta en común con el docente para el armado de la red. Esta actividad tan medular desde el punto de vista de la enseñanza no puede realizarse desde la consigna de construir mapas, porque en ellos cualquier alternativa semántica es válida. Por ejemplo,

Tabla I

Relación entre la calificación obtenida en la evaluación (Anexo I) y el número de alumnos ordenados según las categorías de diseño tipo redes o tipo mapas conceptuales.

\begin{tabular}{|c|c|c|c|}
\hline $\begin{array}{c}\text { Rango de } \\
\text { calificaciones }\end{array}$ & $\begin{array}{c}\text { Diseño tipo red } \\
\text { (Fig. 1) }\end{array}$ & \multicolumn{2}{|c|}{$\begin{array}{c}\text { Diseño tipo mapa } \\
\text { (Fig. 2) }\end{array}$} \\
\hline $10-8$ & 13 & 2 & 1 \\
\hline $7,90-6$ & 7 & 3 & 1 \\
\hline $5,90-4$ & 4 & 4 & \\
\hline $3,90-1$ & 1 & 1 & 2 alumnas \\
& $\begin{array}{c}25 \text { alumnos } \\
\text { en 5 grupos }\end{array}$ & $\begin{array}{c}\text { en 2 grupos } \\
\end{array}$ &
\end{tabular}


podrían escribirse, con diseño de mapa, cualquiera -o todas- las definiciones-resumen de la figura 3 , y la discusión sólo se centraría en cuáles son los conceptos más abarcativos para ubicarlos jerárquicamente en la hoja, de arriba hacia abajo, en el caso de haberse solicitado la construcción de un mapa conceptual.

\section{Aportes de la «discusión en grupo» en la construcción de las redes conceptuales}

Es interesante destacar que «las discusiones en los pequeños grupos para armar la red conceptual se centran en encontrar la forma óptima de redactar las oraciones nucleares para relacionar conceptos»(Galagovsky y Ciliberti, 1994). Esta exigencia conduce a revisar cuáles son los pares de conceptos que se vinculan mejor y a descubrir conceptos subsumidos, que sólo al ser explicitados permiten la correcta vinculación de todos los conceptos. Para ilustrar este punto obsérvese la diferente vinculación entre los conceptos de velocidad, aceleración,fuerza y movimiento que se indica en cada uno de los ejemplos mostrados en las figuras 1, 2 y 3 y en el anexo II. También resulta instructivo de este punto comparar las vinculaciones entre los conceptos de peso y masa en cada uno de esos instrumentos.

En cambio, las discusiones para confeccionar los mapas conceptuales se centran en consensuar sobre el carácter más abarcativo o más inclusivo de los conceptos, para ordenarlos en la forma jerárquica que exige el mapa. Y entendemos que, dado que la jerarquía es dependiente del contexto, la discusión estaría centrada en acuerdos temporales que podrían tener que ver con la imposición de uno de los participantes del grupo por sobre de los otros. Estas discusiones sobre la jerarquía gráfica no nos parecen cuestiones conceptuales interesantes para emplear tiempo de clase en ellas.

La discusión grupal para el armado de las oraciones nucleares de una red conceptual favorece la argumentación en defensa de distintas posibilidades semánticas y esto constituye un importante contenido procedimental (Coll, 1994) que favorece metodologías de aprendizaje más autómomas.

Los momentos de puesta en común para el armado consensuado de la red entre el docente y los alumnos -que en este caso no se hizo para poder realizar la presente experiencia- aporta además otra instancia para señalar y hacer surgir a la luz conflictos cognitivos y facilitar la toma de conciencia de los mismos por los alumnos. Esta discusión general trabajaría, de forma consciente, la resolución del conflicto, pero ya no sería el docente quien lo plantearía para «hacer caer» a los alumnos que no comprendieron el tema, sino que el docente adoptaría una posición más objetiva de señalar contradicciones, redes incompletas, oraciones ambigüas o conexiones incorrectas.
El caso de las dos alumnas que presentaron el mapa 2 y el texto de la figura 3 parece muy representativo de la no discusión con el grupo. El hecho de que ellas no cumplieran con la consigna nos revela no sólo una resistencia -o un problema- actitudinal, sino también su consecuencia, que se refleja en la tarea producida: nos inclinamos a pensar que las alumnas no pudieron armar las oraciones nucleares porque su forma de estudio es fuertemente memorística y tienen serias dificultades para operar en niveles metacognitivos.

Los momentos de discusión grupal ayudan a disgregar estas formas consolidadas de estrategias memorísticas de aprendizaje.

En base a lo observado en nuestra experiencia aquí relatada puede resumirse que:

- Las redes conceptuales son un instrumento que permite, por un lado, indagar mejor que los mapas sobre el aprendizaje conceptual realizado por los alumnos; y, por otro lado, las discusiones grupales que se generan en torno a la elección de las oraciones nucleares más convenientes activan importantes contenidos procedimentales.

- Las de redes conceptuales producidas por los alumnos -sin haber llegado a un consenso con toda la clase-, conjuntamente con las notas que obtienen los alumnos en sus evaluaciones tradicionales, son dos parámetros complementarios que permiten discriminar el rendimiento escolar proveniente de niveles de comprensión o proveniente de aprendizajes memorísticos o de aplicación mecánica de algoritmos de resolución de problemas.

\section{AGRADECIMIENTOS}

Este trabajo ha sido desarrollado gracias al subsidio UBACyT EX-083.

Agradecemos también la colaboración de Marie Christine Aguayo por el armado de mapas y redes conceptuales por ordenador.

\section{NOTAS}

${ }^{1}$ Una oración nuclear es una oración precisa y de máximo significado; por ello, verbos tales como «se relaciona con», «modifica a», «influye sobre», «afecta a», etc. son ambiguos -de hecho la misma raya que une los nodos indica que existe una relación- y no conducen a la elaboración de una oración nuclear.

${ }^{2}$ Esta agrupación fue permitida por tratarse de una experiencia de aula y no de una situación de rigurosa investigación. 


\section{REFERENCIAS BIBLIOGRÁFICAS}

ABELEDO. J. etal. (1992). Física, Unidad2 Dinámica. Argentina: UBA.

COLL, C. et al. (1994). Los contenidos en la reforma. Enseñanza y aprendizaje de conceptos, procedimientos y actitudes. Madrid: Santillana.

CASTIGLIONI, R., PERAZZO, O. y RELA, A. (1994). Física I. Argentina: Troquel.

CHEBLI MURAD, A. (1984). Curso preparatorio para la Universidad Tecnológica Nacional, Regional. Buenos Aires, Argentina.

CHOMSKY,N. (1986). El lenguaje y el entendimiento. España: Seix Barral.

COLÁS BRAVO, M.P. y BUENDÍA EISMAN, L. (1994). Investigación educativa. España: Alfar.

GALAGOVSKY, L.R. (1993a). Redes conceptuales: su base teórica e implicancias para el proceso de enseñanza-aprendizaje de las ciencias, Enseñanza de las Ciencias, 11, pp. 301-307.

GALAGOVSKY, L.R., (1993b). Hacia un nuevo rol docente. Una propuesta diferente para el trabajo en clase. Argentina: Troquel.

GALAGOVSKY, L.R. (1996). Redes conceptuales. Aprendizaje, comunicación y memoria. Argentina: Lugar Editorial.
GALAGOVSKY, L.R. y CILIBERTI, N. (1994). Redes conceptuales: su aplicación como instrumento didáctico en temas de física. Enseñanza de las Ciencias, 12, pp. 338-349.

HEIMLICH, J.E. y PITTELMAN, S.D. (1991). El mapa semántico. Argentina: Aique.

NOVAK, J. y GOWIN, B. (1988). Aprendiendo a aprender. España: Martínez Roca.

NOVAK, J. (1991). Ayudar a los alumnos a aprender cómo aprender. Enseñanza de las Ciencias, 9(3), pp. 215-228.

ONTORIA, A. (1995). Mapas conceptuales. Una técnica para aprender, $5^{\mathrm{a}}$ ed. España: Narcea.

ONTORIA, A. (1995). Los mapas conceptuales y su aplicación en el aula. Argentina: Magisterio del Río de la Plata.

PROCIENCIA (1988). Física. El movimiento 2, volumen I. Argentina: Conicet.

RESNICK, R. y HALLIDAY, D. (1984). Física, parte 1. México: Continental.

TIPLER, P. (1984). Física. Argentina: Reverté.

TRICÁRICO, H. y BAZO, R. Física 4. Argentina: A-Z editora.

[Artículo recibido en abril de 1997 y aceptado en junio de 1998.] 


\section{ANEXO I}

Instrumento evaluativo tomado al curso de 37 alumnos de $4^{\circ}$ año del bachillerato.

\section{Problema 1}

Un ascensor pesa $16.000 \mathrm{~N}$.

a) Calcular la tensión del cable si se eleva con una aceleración dirigida hacia arriba de 1,96 m/s².

b) ¿Cuál es la tensión del cable si el ascensor desciende con una velocidad constante de $2 \mathrm{~m} / \mathrm{s}$ ?

c) ¿Cuál es la tensión del cable si el ascensor está detenido? Señalar en cada caso qué fuerzas actúan sobre el ascensor.

\section{Problema 2}

El sistema de la figura se mueve con una aceleración de $4 \mathrm{~m} / \mathrm{s}^{2}$ y es despreciable el rozamiento entre el suelo y las cajas. Si la masa de la caja 1 es de $10 \mathrm{~kg}$ y el de la caja 2 es de $5 \mathrm{~kg}$ :

a) Realizar el diagrama de cuerpo libre correspondiente a cada caja.

b) Indicar cuál de las fuerzas indicadas en el punto $a$ forman par de interacción.

c) Calcular el valor de la fuerza $F$ aplicada.

d) Hallar el valor de la fuerza que la caja 1 ejerce sobre la caja 2.

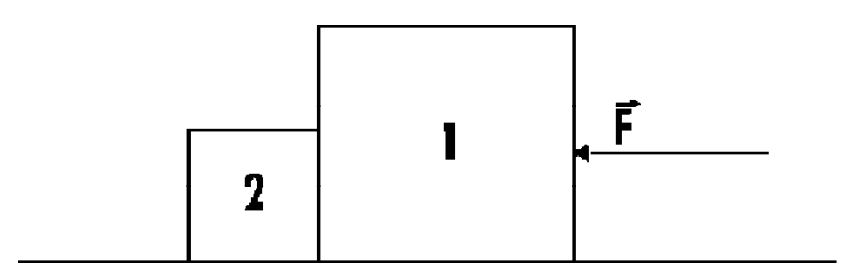




\section{ANEXO II}

Red conceptual sobre el tema de dinámica.

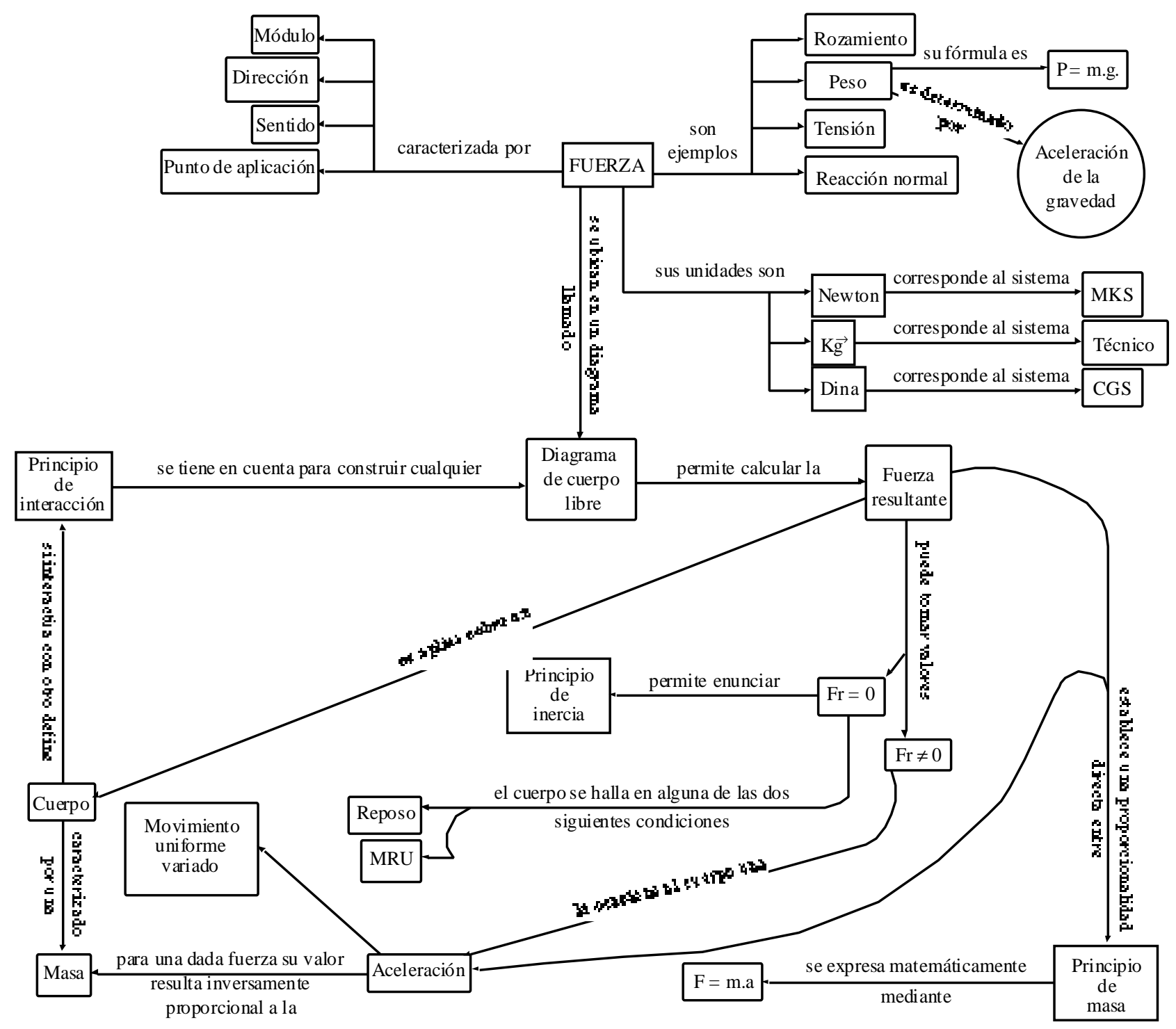

